\title{
MEK/ERK pathway protects ionizing radiation-induced loss of mitochondrial membrane potential and cell death in lymphocytic leukemia cells
}

\author{
T Shonai ${ }^{1}$, M Adachi $^{\star 2}$, K Sakata ${ }^{1}$, M Takekawa ${ }^{3,4}$, T Endo ${ }^{2}$, \\ $\mathrm{K}$ Imai $^{2}$ and $M$ Hareyama ${ }^{1}$ \\ 1 Department of Radiology, Sapporo Medical University School of Medicine, \\ S-1, W-16, Chuo-ku, Sapporo, 060-8543, Japan \\ 2 First Department of Internal Medicine, Sapporo Medical University School of \\ Medicine, S-1, W-16, Chuo-ku, Sapporo, 060-8543, Japan \\ 3 Division of Molecular Cell Signaling, Institute of Medical Science, The \\ University of Tokyo, 4-6-1, Shirokanedai, Minato-ku, Tokyo 108-8639, Japan \\ 4 PRESTO, Japan Science and Technology Corporation (JST), Kawaguchi, \\ Saitama 332-0012, Japan \\ * Corresponding author: M Adachi, The First Department of Internal Medicine, \\ Sapporo Medical University School of Medicine, S-1, W-16, Chuo-ku, Sapporo \\ 060-8543, Japan. Tel: 81-11-611-2111 (3213); Fax: 81-11-611-2282; \\ E-mail: adachi@sapmed.ac.jp
}

Received 2.10.01; revised 28.2.02; accepted 5.3.02

Edited by $\mathrm{H}$ Ichijo

\begin{abstract}
MEK/ERK-mediated signals have recently been found to inhibit Fas-mediated cell death through inhibition of caspase8 activity. It remains unknown whether MEK/ERK-mediated signals affect ionizing radiation (IR)-induced cell death. Here we demonstrate that MEK/ERK-mediated signals selectively inhibit IR-induced loss of mitochondrial membrane potential $\left(\Delta \Psi_{\mathrm{m}}\right)$ and subsequent cell death. In Jurkat cells, TPA strongly activated ERK and inhibited the IR-induced caspase-8/Bid cleavage and the loss of $\Delta \Psi_{m}$. The inhibitory effect of TPA was mostly abrogated by pretreatment of a specific MEK inhibitor PD98059, indicating that the effect depends upon MEK/ERKmediated signals. Moreover, BAF-B03 transfectants expressing IL-2 receptor (IL-2R) $\beta$ C chain lacking the acidic region, which is responsible for MEK/ERK-mediated signals, revealed higher sensitivity to IR than the transfectants expressing wildtype IL-2R. Interestingly, the signals could neither protect the $\Delta \Psi_{m}$ loss nor cell death in UV-irradiated cells. These data imply that the anti-apoptotic effect of MEK/ERK-mediated signals appears to selectively inhibit the IR-induced cell death through protection of the $\Delta \Psi_{m}$ loss. Our data enlighten an antiapoptotic function of MEK/ERK pathway against IR-induced apoptosis, thereby implying its contribution to radioresistance.

Cell Death and Differentiation (2002) 9, 963-971.doi: 10.1038/ sj.cdd. 4401050
\end{abstract}

Keywords: caspase-8; Bid; mitochondrial membrane potential; MEK; ERK; radiosensitivity
Abbreviations: UV, ultravioletlight; $\Delta \Psi_{\mathrm{m}}$, mitochondrial membrane potential; z-IETD-FMK, z-Ile-Glu(OMe)-Thr-Asp(OMe)- $\mathrm{CH}_{2} \mathrm{~F}$; zAAD-CMK, z-Ala-Ala-Asp- $\mathrm{CH}_{2} \mathrm{Cl}$; TPA, Phorbol-12-myristate-13acetate

\section{Introduction}

A variety of intracellular signalings are activated by ionizing radiation (IR), which in turn leads to growth arrest and/or apoptosis. ${ }^{1,2}$ IR-mediated DNA damage elevates p53 activity, and induces pro-apoptotic Bcl-2 family members Bax, Noxa, and PUMA, and a novel p53-inducible molecule AIP1. ${ }^{2-6}$ Once these molecules are induced, they are targeted to the mitochondria and disrupt the mitochondrial membrane potential $\left(\Delta \Psi_{\mathrm{m}}\right)$, which is mediated by opening of the mitochondrial permeability transition (PT) pore. $^{7-9}$ Loss of $\Delta \Psi_{\mathrm{m}}$ subsequently activates executioner caspases-3/9, and eventually leads to cell death. ${ }^{10}$

Human T leukemia Jurkat cells lack p53 ${ }^{\mathrm{WT}}$ functions, and it is thus unlikely that p53-inducible pro-apoptotic molecules contribute in a major way to the IR-induced loss of $\Delta \Psi_{\mathrm{m}}$. In this context, Bid appears to be a candidate as a key molecule inducing the apoptosis, since the cleaved Bid is translocated to the mitochondria and disrupts $\Delta \Psi_{\mathrm{m} \cdot}{ }^{11,12}$ Considering the previous findings that Bid is a substrate of caspase- 8 and DNA damage strongly activates caspase$8,^{11-14}$ caspase-8 may be responsible for IR-mediated Bid cleavage and loss of $\Delta \Psi_{\mathrm{m}}$. However, recent reports suggest that caspase- 8 activation is a marginal event for IR-induced apoptosis, since caspase-8 $(-/-)$ cells had only slightly reduced sensitivity to $\mathrm{IR}^{15}$ and overexpression of a caspase-8 inhibitor, FLIP, did not reduce radiosensitivity. ${ }^{16}$ Thus, it is likely that caspase- 8 is marginal for IR-induced apoptosis.

Recently, it has been found that Fas-L/Fas-mediated death signal is abrogated by the mitogen-activated protein kinase (MAPK/ERK)-mediated signals. ${ }^{17}$ In the Fasmediated apoptotic process, ERK activity inhibits the caspase-8/Bid cleavage, whereas the assembly of a functional death-inducing signaling complex (DISC) is not affected. $^{18}$ This strongly suggests that ERK-mediated signals can suppress apoptosis independently of the Fasmediated signal and the source of this anti-apoptotic activity may exist at the level of caspase-8/Bid activation. This raises the possibility that ERK-mediated signals cannot suppress IR-induced apoptosis, if caspase-8/Bid activation is marginal for the IR-induced apoptotic process. We thus investigated whether TPA-mediated ERK activation can protect Jurkat cells from IR-induced apoptosis. We report here that MEK/ERK-mediated signals can unexpectedly protect Jurkat cells from IR-induced apoptosis, i.e., the 
signals inhibited caspase-8, $-3 /$ Bid activation, the $\Delta \Psi_{m}$ loss, and cell death. However, inhibition of caspase-8/Bid activation alone could not protect Jurkat cells from either cell death or the $\Delta \Psi_{\mathrm{m}}$ loss, though caspase $3 / 8$ activation and DNA fragmentation were substantially inhibited by a caspase inhibitor. In addition, BAF-B03 transfectants A15, which cannot introduce MEK/ERK-mediated signals, revealed higher sensitivity to IR than control F7 transfectants. Thus, the anti-apoptotic effect of MEK/ERK-mediated signals appears to be due to inhibition of the $\Delta \Psi_{m}$ loss in IR-exposed cells, implying that MEK/ERK triggers an antiapoptotic signal discriminating from its inhibitory signal against caspase-8/Bid activation. Furthermore, we show that the MEK/ERK-mediated signals cannot inhibit the UVinduced apoptosis. Taken together, our data imply an unidentified anti-apoptotic action of MEK/ERK-mediated signals which lies upstream of the $\Delta \Psi_{\mathrm{m}}$ loss and selectively contributes to IR-exposed cells.

\section{Results}

\section{Apoptosis in Jurkat cells exposed to IR}

Human T leukemia Jurkat cells are highly sensitive to IR. At $18 \mathrm{~h}$ after exposure to 10-40 Gy-irradiation, Jurkat cells substantially lost cell viability in a dose-dependent manner (Figure 1A). At $12 \mathrm{~h}$ after exposure to IR, low-molecular weight DNA revealed extensive DNA fragmentation by more than 20 Gy-irradiation (Figure 1B). After exposure to 20 Gyirradiation, cell death began to be visible at $12 \mathrm{~h}$ and further increased in a time-dependent manner (Figure 1C). At $6 \mathrm{~h}$ after exposure to 20 Gy-irradiation, breakdown of $\Delta \Psi_{\mathrm{m}}$ appeared and reached near to its highest level at $12 \mathrm{~h}$ (Figure 1D). Phosphatidylserine externalization (PE) is known to occur in apoptotic cells at an early stage and thus we compared the time-course of the $\Delta \Psi_{\mathrm{m}}$ breakdown with that of PE. In Jurkat cells exposed to 20 Gy-irradiation, the timecourses of breakdown of $\Delta \Psi_{\mathrm{m}}$ and PE were quite similar (Figure 1E). We next investigated caspase activation in IRinduced apoptosis. Among a variety of pro-apoptotic signals, $\mathrm{Bcl}-2$ family member Bid, a substrate of caspase-8, can move to mitochondrial membrane and contributes to the opening of the PT pore when Bid is cleaved by caspase-8. ${ }^{12}$ Thus, activation of caspase-8/Bid may be responsible for IRinduced apoptosis upstream of $\Delta \Psi_{\mathrm{m}}$ breakdown. At $6 \mathrm{~h}$ after exposure to 20 Gy-irradiation, distinct cleavage of caspase-8 was observed in Jurkat cells and the cleaved form of caspase8 progressed in a time-dependent manner. Simultaneously, cleavage of caspase-3 and Bid was visible at $6 \mathrm{~h}$ after exposure (Figure 1F). These results indicate that IR induced the $\Delta \Psi_{\mathrm{m}}$ breakdown as an early event during the apoptotic process in Jurkat cells, and that the breakdown began at $6 \mathrm{~h}$ after irradiation together with a variety of other apoptotic events.

\section{Inhibition of caspase activation cannot block IR-induced cell death}

As shown in Figure 1, the time-courses of caspase activation and cleavage of Bid suggest that the overall apoptotic
A

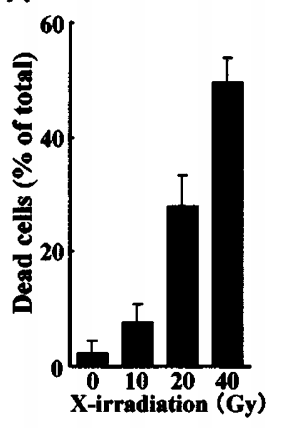

B

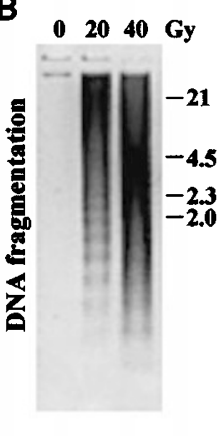

C

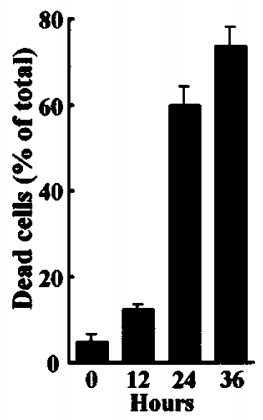

D

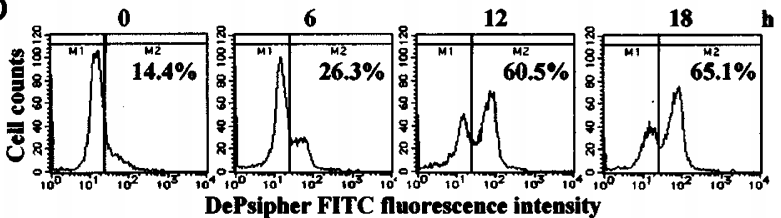

E

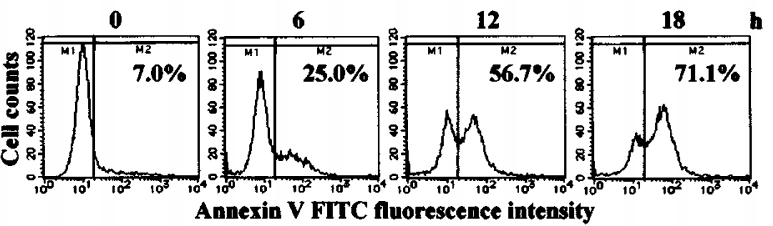

$\mathbf{F}$

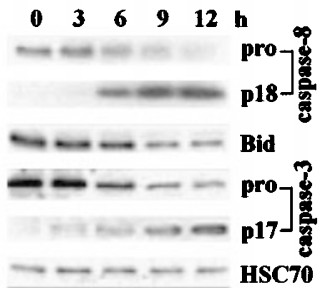

Figure 1 Induction of apoptosis in IR-exposed Jurkat cells. (A) A dosedependent cell death. Cell death was assessed by Trypan blue exclusion assay at $18 \mathrm{~h}$ after exposure to the indicated dose of IR. The columns display the mean standard deviation (S.D.) of data from three separate experiments. (B) DNA fragmentation of low-molecular weight DNA prepared from the cells at $12 \mathrm{~h}$ after exposure to the indicated doses of IR. Molecular weight marker $(\mathrm{kb})$ is shown. (C) Time-course of cell death after 20 Gy-irradiation. Cell viability was evaluated at the indicated hours. (D) The disruption of $\Delta \Psi_{\mathrm{m}}$ at the indicated hours after exposure to $20 \mathrm{~Gy}$-irradiation. Using a DePsipher ${ }^{(i m}$ kit, the $\Delta \Psi_{\mathrm{m}}$ was measured and is shown in histogram plot of FL-1 fluorescence intensity. The percentages express rates of the $\Delta \Psi_{\mathrm{m}}$ loss. (E) PE at the indicated hours after 20 Gy-irradiation. Annexin V-binding capability was evaluated using an ApoAlert Annexin V-FITC kit and is shown in a histogram plot of FL-1 fluorescence intensity. (F) Cleavage of caspases and Bid at the indicated hours after 20 Gy-irradiation. Their cleavage was determined by immunoblotting with the indicated antibodies. HSC70 protein levels show the same amount of protein loaded in each lane

machinery began approximately at $6 \mathrm{~h}$ after exposure to 20 Gy-irradiation in Jurkat cells. We next explored whether caspase-8/Bid activation is upstream of the $\Delta \Psi_{m}$ breakdown. To inhibit caspase-8 activity, we used a cell permeable caspase-8 inhibitor, z-IETD-FMK. Jurkat cells were preincubated with the indicated doses of z-IETD-FMK for $30 \mathrm{~min}$ and then exposed to $20 \mathrm{~Gy}$-irradiation. At $9 \mathrm{~h}$ after irradiation, z-IETD-FMK strongly inhibited elevation of both caspase- 8 and -3 activity in a dose-dependent manner (Figure 2A). The inhibitory effect of z-IETD-FMK was specific, since a Granzyme B inhibitor z-AAD-CMK did not affect their 
A

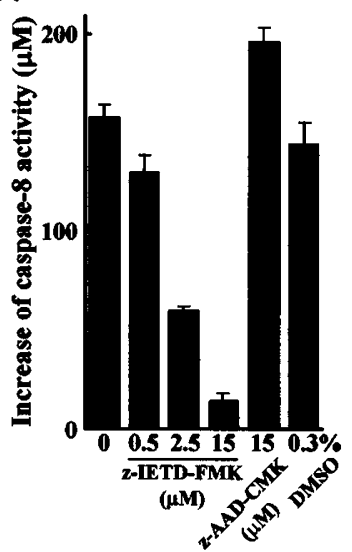

B

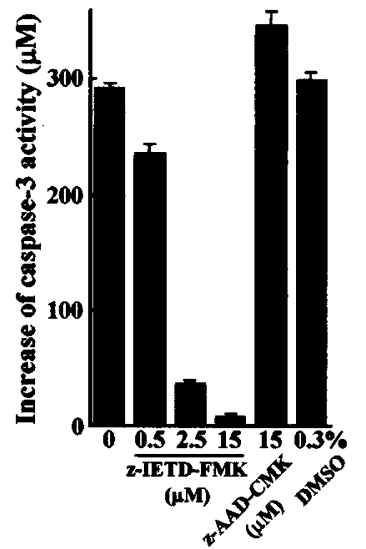

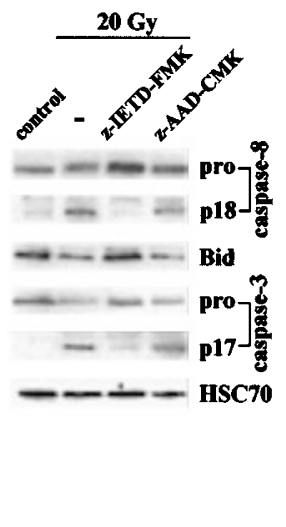

Figure 2 Effect of caspase inhibitor on caspase 3/8 and Bid activation. (A) Jurkat cells were incubated with the indicated concentrations of z-IETD-FMK (a caspase-8 and Granzyme B inhibitor), z-AAD-CMK (a Granzyme B inhibitor) or $0.3 \%$ DMSO (solvent) 30 min before exposure to 20 Gy-irradiation. Caspase-8 and -3 activities were measured at $9 \mathrm{~h}$ after IR-exposure using Caspase- 8 and Caspase- 3 colorimetric protease assay kits, respectively. Each column displays the mean \pm S.D. of data, from which data of unirradiated control cells were subtracted, from three separate experiments. (B) Cells were incubated with $15 \mu \mathrm{M} z-\mathrm{IETD}$ FMK or $15 \mu \mathrm{M}$ z-AAD-CMK 30 min before exposure to 20 Gy-irradiation. At $9 \mathrm{~h}$ after irradiation, cleavage of caspases and Bid was detected by Western blots. HSC70 protein levels show the same amount of protein loaded in each lane

activity. We also confirmed the inhibitory effect of z-IETD-FMK on cleavage of caspase-8, Bid and caspase-3 (Figure 2B). Thus, addition of z-IETD-FMK can suppress IR-induced caspase $3 / 8$ and Bid activation.

We further investigated the effect of z-IETD-FMK on DNA fragmentation. DNA fragmentation is known to depend upon activation of a caspase-activated deoxyribonuclease, $C A D$, which is activated by an active form of caspase3. ${ }^{19,20}$ Since $z$-IETD-FMK can strongly inhibit IR-induced caspase activation, as shown in Figure 2, including caspase-8 and -3, DNA fragmentation may be suppressed. In fact, this inhibitor substantially suppressed DNA fragmentation in 20 Gy-irradiated Jurkat cells (Figure 3A), however it only slightly reduced cell death induced by 20 Gy-irradiation (Figure 3B). Thus, inhibition of both caspase activation and subsequent DNA fragmentation seems not to be sufficient for protection from IR-induced cell death. We next investigated whether Z-IETD-FMK can inhibit the $\Delta \Psi_{\mathrm{m}}$ breakdown. Interestingly, despite its strong inhibitory effect on caspase activation, z-IETD-FMK could not inhibit the IRinduced $\Delta \Psi_{\mathrm{m}}$ breakdown (Figure $3 \mathrm{C}$ ) or the destruction of mitochondrial structure (Figure 3D). From these data, we confirmed that although caspase- 8 and Bid are clearly activated by IR, their contribution to IR-induced apoptosis is marginal. In addition, $\Delta \Psi_{\mathrm{m}}$ appears to be disrupted independently of caspase-8/Bid activation, and the $\Delta \Psi_{\mathrm{m}}$ loss may be crucial in IR-induced cell death.

\section{TPA inhibits IR-induced apoptosis}

The ERK pathway, a major cascade of TCR-mediated signals, ${ }^{21}$ is known to protect $T$ cells from Fas-mediated apoptosis through inhibition of caspase-8/Bid activation. ${ }^{18} \mathrm{We}$ thus explored whether activation of ERK affects IR-induced apoptosis. More than $20 \mathrm{nM}$ TPA preincubation clearly protected Jurkat cells from the apoptosis (Figure 4). When Jurkat cells were incubated with $100 \mathrm{nM}$ TPA $1 \mathrm{~h}$ before exposure to 20 Gy-irradiation, TPA clearly inhibited activation of caspase-8, Bid and caspase-3 (Figure 4B). The antiapoptotic activity of TPA became stronger as the preincubation periods of TPA became more prolonged, and the longest pre-incubation ( $12 \mathrm{~h}$ ) reduced IR-induced cell death to only $15.4 \%$ in irradiated cells without TPA treatment (Figure 4C). Similarly, DNA fragmentation was also inhibited to a greater extent as addition of TPA became earlier (data not shown). Incubation of Jurkat cells with $20 \mathrm{nM}$ TPA at increasingly earlier times before exposure to 40 Gy-irradiation produced a correspondingly stronger inhibition of cleavage of caspase-8, Bid and caspase-3 (Figure 4D). To explore the possible mechanism for the anti-apoptotic effect of TPA against IR-induced apoptosis, we investigated expression levels of anti-apoptotic molecules. Since its anti-apoptotic activity becomes stronger as pre-incubation becomes longer, TPA is supposed to induce some anti-apoptotic molecules. In this context, we investigated expression levels of several antiapoptotic molecules, FLIP, IAP-1 and Bcl-2 in the presence or absence of TPA. However, the treatment did not increase expression levels of these molecules in our experiments (Figure 4E). Thus, TPA-mediated signals can inhibit IRinduced apoptotic cell death independently of induction of FLIP, IAP1 or Bcl-2. Importantly, $20 \mathrm{nM}$ TPA treatment was sufficient to inhibit the IR-induced mitochondrial damage strongly (Figure 4F).

\section{Anti-apoptotic effect of TPA depends upon MEK/ERK pathway}

We next examined whether anti-apoptotic effect of TPA is mediated through MEK/ERK-mediated signals. When Jurkat cells were incubated with $20 \mathrm{nM}$ TPA, ERK was transiently phosphorylated, and this phosphorylation was substantially inhibited by pre-treatment with the specific MEK inhibitor, PD98059. More than $2 \mu \mathrm{M}$ PD98059 can strongly inhibit the ERK phosphorylation (Figure $5 A$ ). Inhibition of MEK activity by 

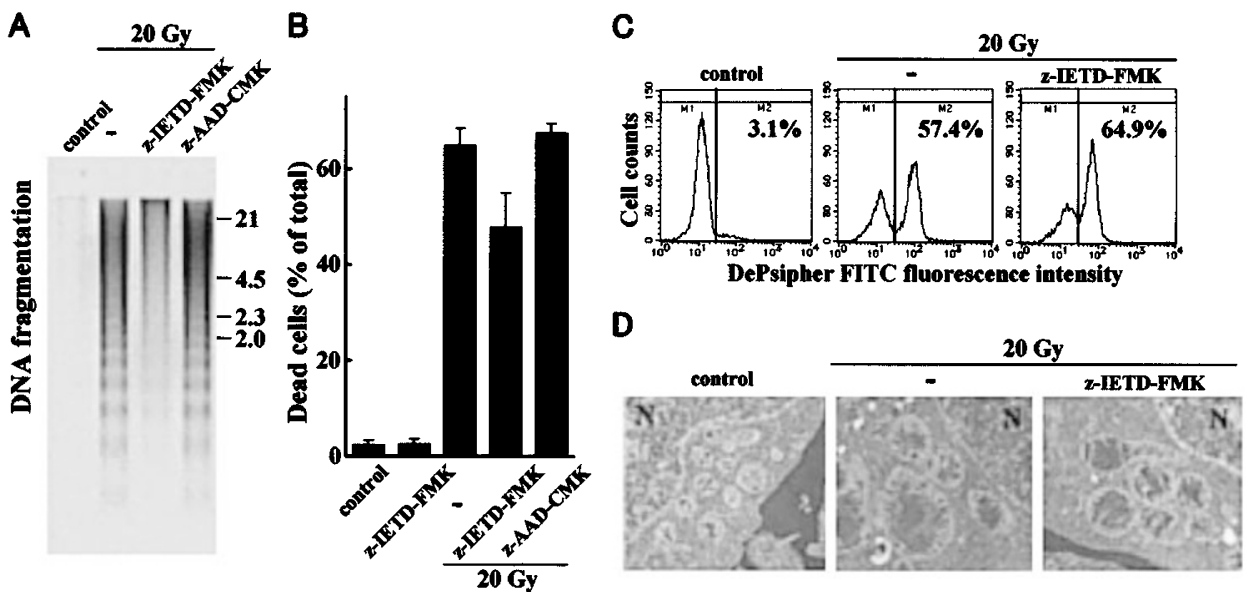

Figure 3 Inhibition of caspase activation was insufficient to suppress IR-induced apoptosis. (A) Effect of Z-IETD-FMK or Z-AAD-CMK on IR-induced DNA fragmentation. Jurkat cells were incubated with each inhibitor $(15 \mu \mathrm{M})$ for $30 \mathrm{~min}$ prior to 20 Gy-irradiation. DNA fragmentation of low-molecular weight DNA prepared from the cells at $12 \mathrm{~h}$ after irradiation. Molecular weight marker $(\mathrm{kb})$ is shown. (B) Effect of z-IETD-FMK or z-AAD-CMK on IR-induced cell death. Cells were incubated with each inhibitor $(15 \mu \mathrm{M})$ for $30 \mathrm{~min}$ prior to $20 \mathrm{~Gy}$-irradiation. At $24 \mathrm{~h}$ after irradiation, cell death was evaluated by Trypan blue exclusion assay and each column displays the mean \pm S.D. of data from three separate experiments. (C) Effect of z-IETD-FMK (15 $\mu \mathrm{M})$ on disruption of $\Delta \Psi_{\mathrm{m}}$. At $12 \mathrm{~h}$ after $20 \mathrm{~Gy}$ irradiation, the $\Delta \Psi_{m}$ was measured by a DePsipher ${ }^{(m)}$ Kit and is shown in a histogram plot of FL-1 fluorescence intensity. The percentages express rates of $\Delta \Psi_{m}$ Ioss. (D) Representative electron micrographs of Jurkat cells with or without incubation of z-IETD-FMK for 30 min prior to 20 Gy-irradiation. The irradiated cells were fixed at $12 \mathrm{~h}$ after exposure. $\mathrm{N}$ refers to nucleus and original magnification is $10000 \times$

PD98059 substantially suppressed the anti-apoptotic effect of TPA on cleavage of caspase-8, Bid, and caspase-3 induced by exposure to $20 \mathrm{~Gy}$-irradiation (Figure 5B). PD98059 also suppressed the anti-apoptotic effect of TPA on PE (Figure $5 \mathrm{C})$. As described above, inhibition of caspase activation alone cannot suppress the IR-induced $\Delta \Psi_{\mathrm{m}}$ breakdown. We thus investigated whether TPA is able to suppress the $\Delta \Psi_{m}$ breakdown. Importantly, TPA treatment was able to strongly suppress an IR-mediated $\Delta \Psi_{\mathrm{m}}$ breakdown to the extent that it was 2.8-fold lower than the control values, but the protective effect was extensively suppressed by pretreatment with PD98059 (Figure 5D). The representative data show the dramatic effect of TPA on $\Delta \Psi_{m}$ to be dependent upon MEK activation and the minimal effect of PD98059 alone (Figure $5 E)$. These data show that MEK/ERK-mediated signals are responsible for the anti-apoptotic effect of TPA on both the caspase activation and the $\Delta \Psi_{\mathrm{m}}$ breakdown in IR-induced apoptosis.

\section{Anti-apoptotic effect of TPA is not found in UV-induced apoptosis}

To examine whether TPA-mediated activation of MEK/ERK pathway protects Jurkat cells from UV-induced apoptosis, we first investigated sensitivity of Jurkat cells to UV irradiation. At $24 \mathrm{~h}$ after exposure to UV $\left(30-90 \mathrm{~J} / \mathrm{m}^{2}\right)$-irradiation, Jurkat cells substantially lost viability in a dose-dependent manner (Figure 6A). UV-irradiation also produced distinct cleavage of Bid and caspase-3 (Figure 6B), and disrupted the $\Delta \Psi_{m}$ (Figure 6C). Thus, Jurkat cells underwent apoptosis by UV irradiation as observed in IR-induced apoptosis. However, in sharp contrast to the strong anti-apoptotic effect of TPA against IR, pre-incubation of $100 \mathrm{nM}$ TPA could not inhibit the UV-induced cell death (Figure 6D), cleavage of Bid and caspase-3 (Figure 6E) or breakdown of $\Delta \Psi_{\mathrm{m}}$ (Figure $6 \mathrm{~F}$ ).
Thus, a MEK/ERK activation-mediated anti-apoptotic effect was selectively observed in IR-induced apoptosis.

\section{Inactivation of MEK/ERK pathway increases radiosensitivity}

We further investigated whether MEK/ERK-mediated signals contribute to radioresistance. To explore this, we used BAFB03 transfectants expressing IL-2R $\beta$ C chain (F7) or its mutant lacking the acidic region (A15). The acidic region is responsible for IL-2-mediated MEK/ERK pathway, but is segregated from anti-apoptotic or proliferation signal pathways, which are believed to require the serine-rich region within the IL-2R $\beta$ c chain. ${ }^{22-24}$ There is thus selective impairment of the MEK/ERK pathway in A15 transfectants when they grow depending upon IL-2. It is noted that there is no distinct difference between F7 and A15 transfectants when they grow in the presence of IL-3. ${ }^{22-24}$ We first investigated phosphorylation levels of ERK after IL-2 stimulation in both transfectants. Prior to IL-2 stimulation, they were cultured in the presence of IL-2 for 7 days and were then deprived of IL-2 for $12 \mathrm{~h}$. The IL-2 stimulation clearly elevated phosphorylation levels of ERK in F7, but not in A15 transfectants (Figure 7A). At $12 \mathrm{~h}$ after exposure to 20 Gy-irradiation, a distinct increase of caspase- 3 was observed in A15, but not in F7 cells (Figure 7B). In addition, 20 Gy-irradiation also disrupted the $\Delta \Psi_{\mathrm{m}}$ in A15, but did not significantly in F7 cells (Figure $7 C$ ). Similarly, the exposure clearly induced cell death in A15, but not in F7 cells at $24 \mathrm{~h}$ after 20 Gy-irradiation (Figure 7D). Importantly, there was no significant difference in the IR-induced cell death between $\mathrm{F} 7$ and $\mathrm{A} 15$ cells when they grew in the presence of IL-3. Thus, reduced MEK/ERK pathway augmented IRinduced $\Delta \Psi_{\mathrm{m}}$ breakdown and subsequent cell death, thereby strongly supporting our idea that MEK/ERK pathway introduces anti-apoptotic signals against IR-induced apoptosis. 
A

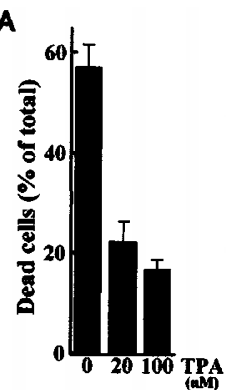

B
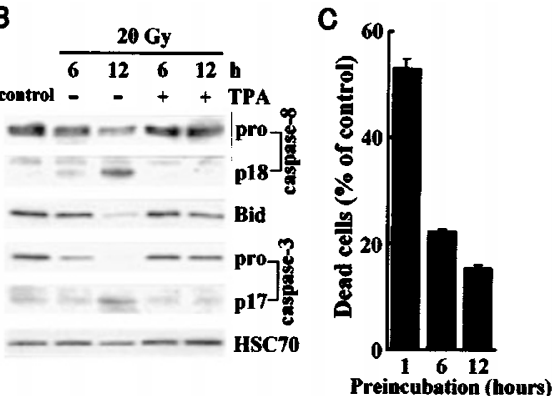

D

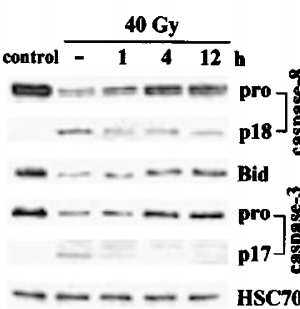

$E$

0136

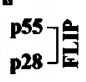

IAP1

$\square=$ Bel-2

$\mathbf{F}$

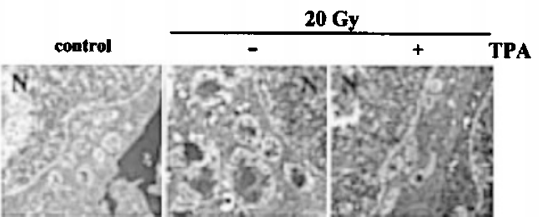

Figure 4 Anti-apoptotic effect of TPA on IR-induced apoptosis. (A) A dosedependent antiapoptotic effect of TPA. Jurkat cells were incubated with the indicated concentrations of TPA for $1 \mathrm{~h}$ prior to $20 \mathrm{~Gy}$-irradiation. At $24 \mathrm{~h}$ after exposure, cell death was evaluated by trypan blue exclusion assay. (B) TPA inhibited cleavage of caspases and Bid during IR-induced apoptosis. Cells were incubated with $(+)$ or without $(-) 100 \mathrm{nM}$ TPA for $1 \mathrm{~h}$ prior to $20 \mathrm{~Gy}$ irradiation. At the indicated periods after exposure, cleavage of the indicated molecules was evaluated by immunoblotting. (C) Augmentation of antiapoptotic effect of TPA by prolonged preincubation. Cells were cultured in the presence of $100 \mathrm{nM}$ TPA for the indicated hours prior to 20 Gy-irradiation. At $24 \mathrm{~h}$ after exposure, cell death was evaluated by trypan blue exclusion assay. Each column displays the mean \pm S.D. of data from three separate experiments (A and C). (D) Augmentation of TPA-mediated suppression of caspases and Bid cleavage by prolonged preincubation. Total cell lysates were prepared at $8 \mathrm{~h}$ after $40 \mathrm{~Gy}$-irradiation. HSC70 protein levels indicate an equal amount of protein loaded in each lane $(B$ and $D)$. (E) No distinct effect of TPA on expression levels of FLIP, IAP-1 or Bcl-2. Total cell lysates $(80 \mu \mathrm{g} /$ lane) were prepared at the indicated periods after incubation with $100 \mathrm{nM}$ TPA and expression levels of the indicated molecules were evaluated by Western blots. (F) Representative electron micrographs of Jurkat cells with $(+)$ or without ( - ) incubation of $20 \mathrm{nM}$ TPA for $1 \mathrm{~h}$ prior to $20 \mathrm{~Gy}$-irradiation. The irradiated cells were fixed at $12 \mathrm{~h}$ after exposure. $\mathrm{N}$ refers to nucleus and original magnification is $10000 \times$

\section{Discussion}

MEK/ERK-mediated signals have recently been found to inhibit caspase-8/Bid activation in Fas-mediated death signals, and protect against subsequent cell death. ${ }^{17,18}$ Here, we demonstrated that MEK/ERK pathway inhibits the IRinduced $\Delta \Psi_{\mathrm{m}}$ breakdown and subsequent cell death in human Jurkat cells, but their suppression occurred independently of caspase-8/Bid cleavage, since inhibition of caspase-8/Bid alone could not protect the $\Delta \Psi_{\mathrm{m}}$ breakdown. In addition, we demonstrated that lack of MEK/ERK activation augmented the IR-induced $\Delta \Psi_{m}$ breakdown and subsequent apoptosis in BAF-B03 transfectants. Thus, our data suggest a novel antiapoptotic function of MEK/ERK-mediated signal which lies upstream of IR-induced breakdown of $\Delta \Psi_{\mathrm{m}}$. Our data also revealed that $\Delta \Psi_{\mathrm{m}}$ breakdown is tightly linked to IR-induced cell death, but activation of caspase- 8 and -3 is marginal for the apoptotic process. Considering recent studies showing that UV-mediated activation of JNK initiates cleavage of Bid, and the tight correlation between the JNK activation and IRinduced apoptosis, ${ }^{25-30} \mathrm{JNK}$-mediated cleavage of Bid and subsequent breakdown of $\Delta \Psi_{\mathrm{m}}$ may be a central pathway for IR-induced apoptosis as observed in UV-mediated apoptosis. However, MEK/ERK-mediated signals could not protect either the breakdown of $\Delta \Psi_{\mathrm{m}}$ or subsequent cell death in UVmediated apoptosis. Thus, IR may induce apoptosis with different machinery by which the $\Delta \Psi_{\mathrm{m}}$ is disrupted independently of caspase-8/Bid, and this process is discriminated by sensitivity to MEK/ERK-mediated signals.

Diverse apoptotic stimuli are believed to transduce signals converging at the mitochondria, which amplifies the death signals by cytochrome $c$ release, and the signals eventually lead to activation of the executioner caspases. ${ }^{31}$ We demonstrated that the breakdown of $\Delta \Psi_{\mathrm{m}}$ is a crucial process in IR-induced apoptosis, but caspase activation may not be. This highlights the importance of the PT pore complex in IR-induced apoptosis. The PT pore complex is mainly composed of voltage-dependent anion channel (VDAC), adenine nucleotide translocator, cyclophilin D and peripheral benzodiazepine receptor. ${ }^{32-34}$ Considering that anti-apoptotic $\mathrm{Bcl}-2$ family members $\mathrm{Bcl}-2$ and $\mathrm{Bcl}-\mathrm{x}_{\mathrm{L}}$ associate with VDAC and close the channel to protect cells from apoptosis, and $\mathrm{Bcl}-2$ clearly suppresses IR-induced apoptosis, ${ }^{34}$ VDAC is a possible candidate as the target of IR. In this context, it is important to clarify the mechanism, by which the $\Delta \Psi_{\mathrm{m}}$ is disrupted during IR-induced apoptosis in p53 $(-/-)$ cells.

We clearly demonstrate that MEK/ERK pathway selectively protects the IR-induced $\Delta \Psi_{\mathrm{m}}$ breakdown. It is obviously important to clarify how MEK/ERK pathway inhibits the $\Delta \Psi_{m}$ breakdown. There are several possible mechanisms. First, MEK/ERK activity could phosphorylate anti-apoptotic or pro-apoptotic proteins directly or indirectly to modulate their functions either positively or negatively, respectively. Recently, TPA was found to stimulate phosphorylation of a pro-apoptotic Bcl-2 family member, $B A D$, at serine 112 through p90Rsk in a PKC-dependent manner, and rescue $T$ lymphocytes from Fas-mediated apoptosis. ${ }^{35}$ Although the contribution of BAD to IR-induced apoptosis remains uncertain, BAD is a good candidate for the MEK/ERK-mediated signals inhibiting IR-induced apoptosis. Second, the signals could induce anti-apoptotic molecules. Among a variety of candidates, the $\mathrm{Bcl}-2$ molecule appears to be a possible likely target of MEK/ ERK-mediated pathway, since Bcl-2 expression level is known to be up-regulated by ERK. ${ }^{36}$ However, in our experiments, TPA did not increase $\mathrm{Bcl}-2$ protein levels significantly (Figure 4E). Furthermore, MEK/ERK activation could not protect the UV-induced $\Delta \Psi_{\mathrm{m}}$ breakdown. Since $\mathrm{Bcl}-2$ can also suppress UV-induced apoptosis, ${ }^{37}$ the induction of anti-apoptotic $\mathrm{Bcl}-2$ family proteins may be unlikely for the anti-apoptotic effect of MEK/ERK-mediated signals. Recently, a variety of proteins have been identified to be targeted to mitochondrial membrane and disrupt $\Delta \Psi_{\mathrm{m}}$ 
A

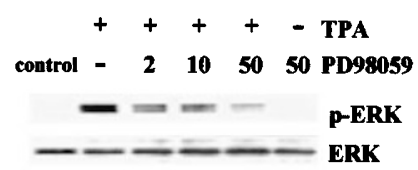

B

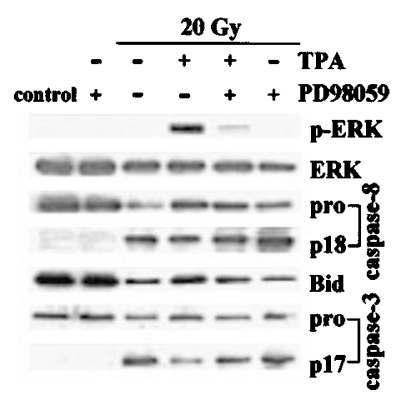

C

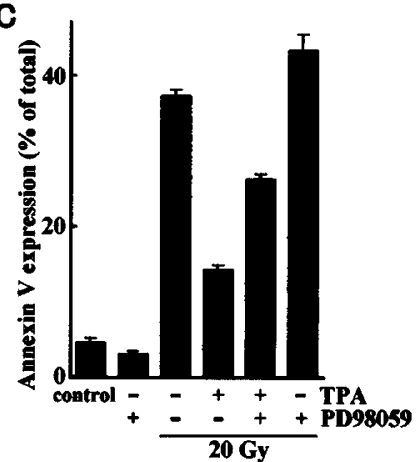

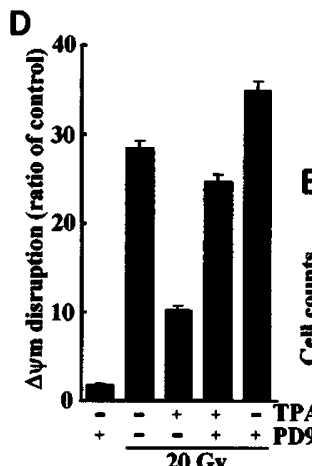

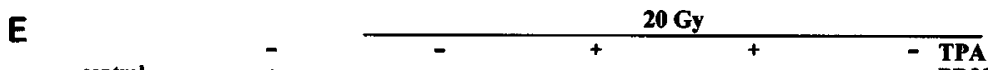

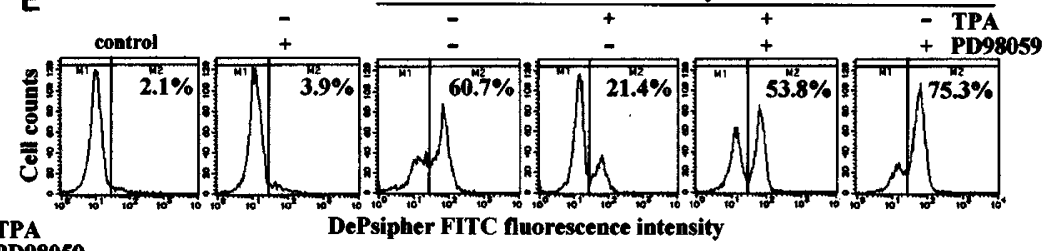

DePsipher FITC fluorescence intensity

$20 \mathrm{~Gy}$

Figure 5 MEK/ERK activation is required for anti-apoptotic effect of TPA against IR-induced apoptosis. (A) A dose-dependent inhibitory effect of a MEK inhibitor, PD98059 on TPA-induced ERK phosphorylation. The indicated doses of PD98059 were added for 30 min prior to incubation with 20 nM TPA and its inhibitory effect was evaluated by decrease of ERK phosphorylation. (B) Inhibitory effect of PD98059 on TPA-mediated protection of caspases and Bid cleavages. Twenty $\mu$ M PD98059 was added for 30 min prior to incubation with $20 \mathrm{nM}$ TPA for $1 \mathrm{~h}$. After incubation with $(+)$ or without $(-)$ the indicated reagents, Jurkat cells were irradiated at 20 Gy and harvested at $9 \mathrm{~h}$ after exposure. The cleavage of caspases and Bid was determined by immunoblotting with the indicated antibodies. HSC70 protein levels show the same amount of protein loaded in each lane. (C) PD98059 inhibits the anti-apoptotic effect of TPA on PE. Cells ware preincubated with $20 \mathrm{nM}$ TPA and/or $20 \mu \mathrm{M}$ PD98059 as experiments in B. PE was evaluated by detection of binding capability to Annexin V at $12 \mathrm{~h}$ after $20 \mathrm{~Gy}$-irradiation, and data from three independent experiments are shown in a histogram plot of FL-1 fluorescence intensity. (D) PD98059 inhibits the TPA-mediated suppression of $\Delta \Psi_{\mathrm{m}}$ loss. Cells were harvested at $15 \mathrm{~h}$ after $20 \mathrm{~Gy}$-irradiation with (+) or without (-) preincubation with $20 \mathrm{nM}$ TPA and/or $20 \mu \mathrm{M}$ PD98059 as experiments in B. Each column displays the mean \pm S.D. of data from three separate experiments. (E) Representative results of $\Delta \Psi_{m}$ in $D$. The percentages express rates of the $\Delta \Psi_{\mathrm{m}}$ loss

in a wide range of apoptotic stimuli. Further studies will reveal a molecule which selectively contributes to the IRinduced $\Delta \Psi_{\mathrm{m}}$ breakdown, and clarify the mechanism for the anti-apoptotic function of MEK/ERK-mediated signals.

Considering the fact that mutations are frequently detected in Ras/Raf signaling molecules upstream of MEK/ERK pathway in primary lymphoid malignancies ${ }^{38}$ resulting in constitutive activation of MEK/ERK signals, their aberrant activation is likely to contribute to radioresistance. In fact, previous studies have showed that overexpression of the activated forms of c-Raf is associated with increased radioresistance. ${ }^{39,40}$ However, there are several reports conflicting those data, ${ }^{41,42}$ thereby implying that MEK/ERK signals may contribute to radiosensitivity differently in a cell type specific manner. In this regard, further clarification of the precise molecular mechanism(s) is required to understand variable radiosensitivity of the malignant cells.

\section{Materials and Methods}

\section{Cell culture}

Human T-cell leukemia Jurkat cells, obtained from the Japanese Cancer Research Resources Bank (Tokyo, Japan), were grown in RPMI supplemented with $10 \%$ fetal calf serum (FCS). Murine IL-3- dependent hematopoietic cell line BAF-B03 transfectants (F7 and A15) were kindly gifted from Dr. T Taniguchi (Tokyo University), and maintained in RPMI supplementaed with $10 \%$ FCS plus $10 \%$ WEHI-3B conditioned medium (as a source of murine IL-3) or human IL-2 (400 IU/ml; kindly provided by Shionogi Chemical Pharmacy, Osaka, Japan). Cells were exposed at room temperature to IR using a $150 \mathrm{kV}$ $\mathrm{X}$-ray equipment (M-150WE, Softex, Tokyo) that can provide a dose rate of approximately $110 \mathrm{cGy} / \mathrm{min}$ or to UV using a $254 \mathrm{~nm}$ UV crosslinker (CL-1000, UV Products, Upland, CA, USA). To evaluate cell viability, cells were mixed with the same volume of $0.4 \%$ Trypan blue solution, and immediately examined to determine whether they can exclude the dye under light microscopical observation.

\section{Materials}

z-IETD-FMK (caspase-8 and Granzyme $B$ inhibitor), z-AAD-CMK (Granzyme B inhibitor), Phorbol-12-myristate-13-accetate (TPA) and PD98059 (a MEK inhibitor, 2'-Amino-3'-methoxyflavone) were obtained from Calbiochem-Novabiochem Co. (Darmstadt, Germany).

\section{Antibodies}

The anti-caspase-3/CPP32 antibody was purchased from BD PharMingen (SanDiego, CA, USA). The anti-caspase-8 and anti-Bid antibodies were from MBL (Nagoya, Japan). The anti-phospho-p42/44 MAP kinase antibody was from NEB (Beverly, MA, USA). The antiERK1 and anti-HSC70 antibodies were from Santa Cruz Biotechnology Inc. (Santa Cruz, CA, USA). 
A

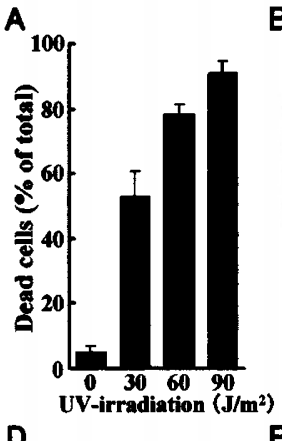

D

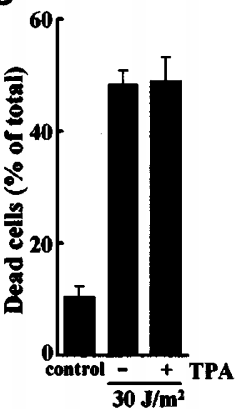

B
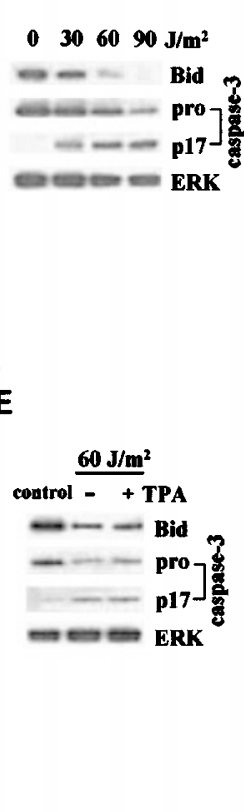

C

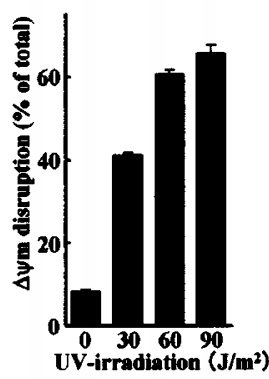

$\mathbf{F}$

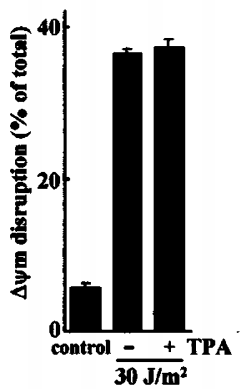

Figure 6 Effect of TPA on apoptosis induced by UV-irradiation in Jurkat cells. (A) A dose-dependent cell death by UV-irradiation. Cell death was assessed by Trypan blue exclusion assay at $24 \mathrm{~h}$ after exposure to the indicated dose of UV-irradiation. (B) Cleavage of Bid and caspase-3 at $9 \mathrm{~h}$ after exposure to the indicated dose of UV-irradiation. (C) Disruption of $\Delta \Psi_{\mathrm{m}}$ at $12 \mathrm{~h}$ after exposure to the indicated dose of UV-irradiation. (D) Cell death assessed by Trypan blue exclusion assay at $24 \mathrm{~h}$ after exposure. The columns display the mean \pm S.D. of data from three separate experiments in A, D. (E) Cleavage of Bid and caspase- 3 at $9 \mathrm{~h}$ after exposure. ERK protein levels show the same amount of protein loaded in each lane in $\mathrm{B}, \mathrm{E}$. (F) Disruption of $\Delta \Psi_{\mathrm{m}}$ at $12 \mathrm{~h}$ after exposure. The $\Delta \Psi_{m}$ is shown in a histogram plot of FL-1 fluorescence intensity. The percentages express rates of the $\Delta \Psi_{m}$ loss from three independent experiments in $\mathrm{C}, \mathrm{F}$. Cells were incubated with $(+)$ or without $(-) 100 \mathrm{nM}$ TPA for $1 \mathrm{~h}$ prior to exposure to UV $\left(30\right.$ or $\left.60 \mathrm{~J} / \mathrm{m}^{2}\right)$ in D, E, F

\section{DNA fragmentation assay}

As described previously, ${ }^{43}$ low-molecular weight genomic DNA extracted with the lysis buffer $(0.5 \%$ Triton X-100, 10 nM EDTA and $10 \mathrm{mM}$ Tris-HCl, $\mathrm{pH}$ 7.4) was treated with $400 \mu \mathrm{g} / \mathrm{ml}$ of RNase A and Proteinase $\mathrm{K}$ for $1 \mathrm{~h}$ at $37^{\circ} \mathrm{C}$, ethanol precipitated and subjected onto $1.0 \%$ agarose gels. The gels were stained with $1 \mu \mathrm{g} / \mathrm{ml}$ of ethidium bromide.

\section{Annexin V-FITC staining assay}

Following the manufacturer's protocol (ApoAlert Annexin $\mathrm{V}$ Apoptosis Kit, Clontech), $1 \times 10^{6}$ cells were incubated with Annexin $V$ at room temperature for $15 \mathrm{~min}$ in the dark. The cells were then analyzed by flow cytometry (FACSCalibur, Becton Dickinson Immnocytometry Systems, San Jose, CA, USA) using a single laser emitting excitation light at $488 \mathrm{~nm}$. The data were converted to histogram (FL1) plots using CellQuest software.

\section{Western blotting}

After washing with ice-cold PBS, cells were lyzed by adding $50 \mu \mathrm{l}$ of RIPA buffer (100 mM NaCl, 2 mM EDTA, 1 mM PMSF, 1\% NP-40 and $50 \mathrm{mM}$ Tris- $\mathrm{HCl}(\mathrm{pH} 7.2))$. Total cell lysates were collected and their protein concentration was evaluated using a Protein Assay (BioRad, Melville, NY, USA). The lysates ( $80 \mu \mathrm{g} /$ lane) were separated by $10-$ $15 \%$ SDS-PAGE gels and then electrophoretically transferred to PVDF membranes (Millipore, Bedford, MA) at $18 \mathrm{~V}$ for $70 \mathrm{~min}$. Membranes were soaked into Block Ace (Dainippon Pharmacia Co., Tokyo) overnight at $4^{\circ} \mathrm{C}$ and washed with the washing buffer $(140 \mathrm{mM}$ $\mathrm{NaCl}, 25 \mathrm{mM}$ Tris- $\mathrm{HCl}(\mathrm{pH} 7.8)$ and $0.05 \%$ Tween 20). The membranes were incubated with primary antibodies overnight at $4^{\circ} \mathrm{C}$, and thereafter incubated with the corresponding peroxidaselinked secondary antibodies (Amersham or MBL) for $1 \mathrm{~h}$ at room temperature. Signals were developed by a standard enhanced chemiluminescence (ECL) method following the manufacturer's protocol (Amersham).
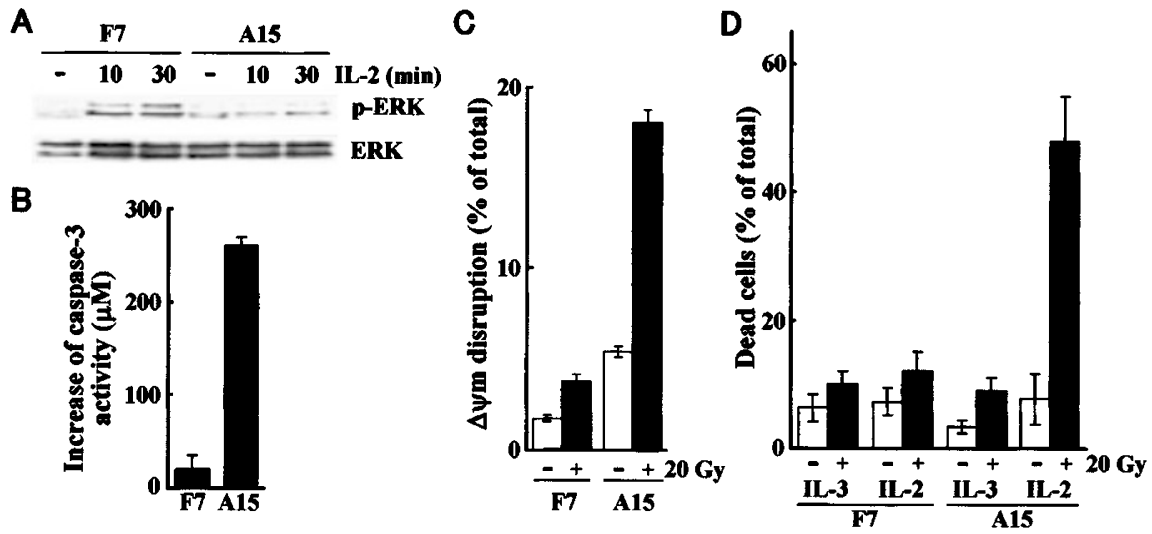

Figure 7 Induction of apoptosis in IR-exposed BAF-B03 transfectants. (A) A15 has no distinct ERK activation in response to IL-2. The proliferating F7 and A15 cells were deprived of IL-2 for $12 \mathrm{~h}$ and thereafter incubated with $400 \mathrm{IU} / \mathrm{ml} \mathrm{IL}-2$ for the indicated minutes. Phosphorylation levels of ERK were evaluated by immunoblotting with anti-phosphorylated ERK specific antibody. ERK protein levels show the same amount of protein loaded in each lane. (B) IR-induced caspase3 activation in F7 and A15 transfectants. F7 and A15 cells were cultured in the presence of IL-2 for 7 days, and caspase- 3 activity was measured at $12 \mathrm{~h}$ after 20 Gyirradiation using a Caspase-3 colorimetric protease assay kit. Each column displays the mean \pm S.D. of data, from which data of unirradiated control cells were subtracted, from three separate experiments. (C) Disruption of $\Delta \Psi_{\mathrm{m}}$ at $18 \mathrm{~h}$ after 20 Gy-irradiation. Prior to 20 Gy-irradiation, $\mathrm{F} 7$ and $\mathrm{A} 15$ cells were cultured in the presence of IL-2 for 7 days, and the $\Delta \Psi_{m}$ is shown in a histogram plot of FL-1 fluorescence intensity. The percentages express rates of the $\Delta \Psi_{m}$ loss. (D) Cell death assessed by Trypan blue exclusion assay at $24 \mathrm{~h}$ after exposure. F7 and A15 cells were cultured in the presence of IL-2 or IL-3 for 7 days prior to 20 Gy-irradiation. Each column displays the mean \pm S.D. of data from three separate experiments in $\mathrm{C}, \mathrm{D}$ 


\section{Caspase- 8 and caspase- 3 colorimetric protease assay}

Following the manufacturer's protocol (FLICE/Caspase-8 and CPP32/ Caspase- 3 Colorimetric Protease Assay Kit, MBL), $2 \times 10^{6}$ cells were lyzed in $250 \mu \mathrm{l}$ of chilled Cell Lysis Buffer and incubated on ice for $10 \mathrm{~min}$. Total cell lysates were collected and their protein concentration was evaluated using a Protein Assay (BioRad). Total cell lysates $(100-200 \mu \mathrm{g})$ were mixed with the same volume of $2 \times$ Reaction Buffer. The mixtures were incubated with the $4 \mathrm{mM}$ IETD-pNA or VETD-pNA Substrate $(200 \mu \mathrm{M})$ at $37^{\circ} \mathrm{C}$ for $1 \mathrm{~h}$. The samples were then analyzed at $400 \mathrm{~nm}$ in a spectrophotometer (Shimadzu, Kyoto, Japan).

\section{Detection of mitochondrial membrane potential disruption}

Following the manufacturer's protocol (DePsipher ${ }^{\mathrm{TM}}$ Kit, Trevigen, Gaithersburg, MD, USA), $1 \times 10^{6}$ cells were suspended in $1 \mathrm{ml}$ of prewarmed DePsipher solution $(5 \mu \mathrm{g} / \mathrm{ml})$, and incubated at $37^{\circ} \mathrm{C}$, in $5 \% \mathrm{CO}_{2}$ for $20 \mathrm{~min}$. After washing with PBS twice, the cells were analyzed by flow cytometry (FACSCalibur, Becton Dickinson) using a single laser emitting excitation light at $488 \mathrm{~nm}$. The data were converted to histogram (FL1) plots using CellQuest software.

\section{Electron microscopy}

Cells were fixed by the addition of the same volume of $2.5 \%$ glutaraldehyde and post-fixed in $1 \%$ osmium tetroxide (TAAB, Berkshire, UK). After fixation, samples were washed in $7.5 \%$ sucrose, stepwise-dehydrated in 50-100\% ethanol, washed again in propylene oxide twice and embedded in epoxy resin (TAAB). Samples were mounted in mesh, stained with uranium acetate and lead citrate, and thereafter mitochondrial structure was observed by Electron microscope (JEM-1200EX, JEOL, Tokyo).

\section{Acknowledgements}

We thank Dr. Tadatsugu Taniguchi (Tokyo University) for the BAF-B03 transfectants (F7 and A15). We are very grateful to Mr. Robert Holmes for help with preparation of this manuscript. Supported by Grants-in-Aid for Cancer Research and for Scientific Research from the Ministry of Education, Science, Sports and Culture of Japan (M Adachi, K Imai, M Hareyama) and the Novartis Foundation for the Promotion of Science (M Adachi).

\section{References}

1. Hartwell LH and WeinertTA (1989) Checkpoints: controls that ensure the order of cell cycle events. Science 246: 629-634

2. Clarke AR, Purdie CA, Harrison DJ, Morris RG, Bird CC, Hooper ML and Wyllie AH (1993) Thymocyte apoptosis induced by p53-dependent and independent pathways. Nature 362: 849-852

3. Kitada S, Krajewski S, Miyashita T, Krajewska M and Reed JC (1996) Gammaradiation induces upregulation of $\mathrm{Bax}$ protein and apoptosis in radiosensitive cells in vivo. Oncogene 12: 187-192

4. Oda E, Ohki R, Murasawa H, Nemoto J, Shibue T, Yamashita T, Tokino T, Taniguchi T and Tanaka N (2000) Noxa, a BH3-only member of the Bcl-2 family and candidate mediator of $p 53$-induced apoptosis. Science 288: 1053-1058

5. Nakano Kand Vousden KH (2001) PUMA, a novel proapoptotic gene, is induced by p53. Mol. Cell 7: 683-694
6. Oda K, Arakawa H, Tanaka T, Matsuda K, Tanikawa C, Mori T, Nishimori H, Tamai K, Tokino T, Nakamura Y and Taya Y (2000) p53AIP1, a potential mediator of p53-dependent apoptosis, and its regulation by Ser-46-phosphorylated p53. Cell 102: 849-862

7. Green DRand ReedJC (1998) Mitochondria and apoptosis. Science 281:13091312

8. Petit PX, Zamzami N, Vayssiere JL, Mignotte B, Kroemer G and Castedo M (1997) Implication of mitochondria in apoptosis. Mol. Cell. Biochem. 174: 185188

9. Matsuyama S, Llopis J, Deveraux QL, Tsien RY and Reed JC (2000) Changes in intramitochondrial and cytosolic $\mathrm{pH}$ : early events that modulate caspase activation during apoptosis. Nat. Cell. Biol. 2: 318-325

10. Li P, Nijhawan D, Budihardjo I, Srinivasula SM, Ahmad M, Alnemri ES and Wang X (1997) Cytochrome $c$ and dATP-dependent formation of Apaf-1/caspase-9 complex initiates an apoptotic protease cascade. Cell 91: 479-489

11. LuoX, Budihardjo I, Zou H, Slaughter Cand Wang X(1998) Bid, a Bcl2 interacting protein, mediates cytochrome $c$ release from mitochondria in response to activation of cell surface death receptors. Cell 94: 481-490

12. Zamzami N, El Hamel C, Maisse C, Brenner C, Munoz-Pinedo C, Belzacq AS, Costantini P, Vieira H, Loeffler M, Molle G and Kroemer G (2000) Bid acts on the permeability transition pore complex to induce apoptosis. Oncogene 19: 63426350

13. Wesselborg S, Engels IH, Rossmann E, Los M and Schulze-Osthoff K (1999) Anticancer drugs induce caspase-8/FLICE activation and apoptosis in the absence of CD95 receptor/ligand interaction. Blood 93: 3053-3063

14. Sheikh MS, Antinore MJ, Huang $Y$ and Fornace Jr AJ (1998) Ultravioletirradiation-induced apoptosis is mediated via ligand independent activation of tumor necrosis factor receptor 1. Oncogene 17: 2555-2563

15. Belka C, Rudner J, Wesselborg S, Stepczynska A, Marini P, Lepple-Wienhues A, Faltin H, Bamberg M, Budach W and Schulze-Osthoff K (2000) Differential role of caspase-8 and BID activation during radiation- and CD95-induced apoptosis. Oncogene 19: 1181-1190

16. Kataoka T, Schroter M, Hahne M, Schneider P, Irmler M, Thome M, Froelich CJ and Tschopp J (1998) FLIP prevents apoptosis induced by death receptors but not by perforin/granzyme B, chemotherapeutic drugs, and gamma irradiation. J. Immunol. 161: 3936-3942

17. Wilson DJ, Alessandrini A and Budd RC (1999) MEK 1 activation rescues Jurkat T cells from Fas-induced apoptosis. Cell Immunol. 194: 67-77

18. Holmstrom TH, Schmitz I, Soderstrom TS, Poukkula M, Johnson VL, Chow SC, Krammer PH and Eriksson JE (2000) MAPK/ERK signaling in activated T cells inhibits CD95/Fas-mediated apoptosis downstream of DISC assembly. EMBOJ. 19: $5418-5428$

19. Enari M, Sakahira H, Yokoyama H, Okawa K, Iwamatsu A and Nagata S (1998) A caspase-activated DNase that degrades DNA during apoptosis, and its inhibitor ICAD. Nature 391: 43-50

20. Sakahira $H$, Enari $M$ and Nagata $S$ (1998) Cleavage of CAD inhibitor in CAD activation and DNA degradation during apoptosis. Nature 391: 96-99

21. Lewis TS, Shapiro PS and Ahn NG (1998) Signal transduction through MAP kinase cascades. Adv. Cancer Res. 74: 49-139

22. Shibuya H, Yoneyama M, Ninomiya-Tsuji J, Matsumoto $\mathrm{K}$ and Taniguchi $\mathrm{T}$ (1992) IL-2 and EGF receptors stimulate the hematopoietic cell cycle via different signaling pathways: demonstration of a novel role for c-myc. Cell 70: $57-67$

23. Miyazaki T, Liu ZJ, Kawahara A, Minami Y, Yamada K, Tsujimoto Y, Barsoumian EL, Permutter RM and Taniguchi T (1995) Three distinct IL-2 signaling pathways mediated by bcl-2, c-myc, and lck cooperate in hematopoietic cell proliferation. Cell 81: 223-231

24. Adachi M, Sekiya M, Torigoe T, Takayama S, Reed JC, Miyazaki T, Minami Y, Taniguchi T and Imai K (1996) Interleukin-2 (IL-2) upregulates BAG-1 gene expression through serine-rich region within IL-2 receptor betacchain. Blood 88: $4118-4123$

25. Tournier C, Hess P, Yang DD, Xu J, Turner TK, Nimnual A, Bar-Sagi D, Jones SN, Flavell RA and Davis RJ (2000) Requirement of JNK for stress-induced activation of the cytochrome c-mediated death pathway. Science 288: 870-874

26. Kharbanda S, Ren R, Pandey P, Shafman TD, Feller SM, Weichselbaum RRand Kufe DW (1995) Activation of the c-Abl tyrosine kinase in the stress response to DNA-damaging agents. Nature 376: 785-788 
27. Yang DD, Kuan CY, Whitmarsh AJ, Rincon M, Zheng TS, Davis RJ, Rakic P and Flavell RA (1997) Absence of excitotoxicity-induced apoptosis in the hippocampus of mice lacking the Jnk3 gene. Nature 389: 865-870

28. Chen YR, Meyer CF and Tan TH (1996) Persistent activation of c-Jun N-terminal kinase 1 (JNK1) in gamma radiation-induced apoptosis. J. Biol. Chem. 271: $631-634$

29. Faris M, Kokot N, Latinis K, Kasibhatla S, Green DR, Koretzky GA and Nel A (1998) The c-Jun N-terminal kinase cascade plays a role in stress-induced apoptosis in Jurkat cells by up-regulating Fas ligand expression. J. Immunol. 160: $134-144$

30. Chen YR, WangX, Templeton D, Davis RJ and Tan TH(1996) The role of C-Jun Nterminal kinase (JNK) in apoptosis induced by ultraviolet $\mathrm{C}$ and gamma radiation. Duration of JNK activation may determine cell death and proliferation. J. Biol. Chem. 271: $31929-31936$

31. Kuwana T, Smith JJ, Muzio M, Dixit V, Newmeyer DD and Kornbluth S (1998) Apoptosis induction by caspase- 8 is amplified through the mitochondrial release of cytochrome c. J. Biol. Chem. 273: 16589-16594

32. Crompton M, Virji S and Ward JM (1998) Cyclophilin-D binds strongly to complexes of the voltage-dependent anion channel and the adenine nucleotide translocase to form the permeability transition pore. Eur. J. Biochem. 258: 729 735

33. Crompton $\mathrm{M}$ (1999) The mitochondrial permeability transition pore and its role in cell death. Biochem. J. 341:233-249

34. Shimizu S, Narita M and Tsujimoto Y (1999) Bcl-2 family proteins regulate the release of apoptogenic cytochrome $\mathrm{c}$ by the mitochondrial channel VDAC. Nature 399: 483-487

35. Bertolotto C, Maulon L, Filippa N, Baier G and Auberger P (2000) Protein kinase $\mathrm{C}$ theta and epsilon promote T-cell survival by a rsk-dependent phosphorylation and inactivation of BAD. J. Biol. Chem. 275: 37246-37250
36. Boucher MJ, Morisset J, Vachon PH, Reed JC, Laine J and Rivard N (2000) MEK ERK signaling pathway regulates the expression of $\mathrm{Bcl}-2, \mathrm{Bcl}-\mathrm{X}(\mathrm{L})$, and $\mathrm{Mcl}-1$ and promotes survival of human pancreatic cancer cells. J. Cell Biochem. 79: $355-369$

37. Reed JC (1994) Bcl-2 and the regulation of programmed cell death. J. Cell. Biol. 124: $1-6$

38. BrowettPJ and Norton JD (1989) Analysis of ras gene mutations and methylation state in human leukemias. Oncogene 4: 1029-1036

39. Cunningham JM, Francis GE, Holland MJ, Pirollo KF and Chang EH (1991) Aberrant DNA topoisomerase II activity, radioresistance and inherited susceptibility to cancer. Br. J. Cancer 63: 29-36

40. Riva C, Lavieille JP, Reyt E, Brambilla E, Lunardi J and Brambilla C (1995) Differential c-myc, c-jun, c-raf and p53 expression in squamous cell carcinoma of the head and neck: implication in drug and radioresistance. Eur. J. Cancer B. Oral. Oncol. 31: 384-391

41. Warenius HM, Browning PG, Britten RA, Peacock JA and Rapp UR (1994) C-raf1 proto-oncogene expression relates to radiosensitivity rather than radioresistance. Eur. J. Cancer 30: 369-375

42. Gupta AK, Bernhard EJ, Bakanauskas VJ, Wu J, Muschel RJ and McKenna WG (2000) RAS-Mediated radiation resistance is not linked to MAP kinase activation in two bladder carcinoma cell lines. Radiat. Res. 154: 64-72

43. Koyama Y, Adachi M, Sekiya M, Takekawa M and Imai K (2000) Histone deacetylase inhibitors suppress IL-2-mediated gene expression prior to induction of apoptosis. Blood 96: 1490-1495 